\title{
A single dose of beetroot juice improves endothelial function but not tissue oxygenation in pregnant women: a randomised clinical trial
}

\author{
Mônica Volino-Souza, Gustavo Vieira de Oliveira and Thiago Silveira Alvares* \\ Nutrition and Exercise Metabolism Research Group, Nutrition Institute, Universidade Federal do Rio de Janeiro, Macaé, \\ 27979-000 Rio de Janeiro, Brazil \\ (Submitted 15 January 2018 - Final revision received 13 June 2018 - Accepted 22 July 2018 - First published online 19 September 2018)
}

\section{Abstract}

Beetroot juice (BJ) consumption has been associated with improved cardiovascular health owing to an increase in NO bioconversion. This study evaluates the effect of BJ consumption on macrovascular endothelial function (flow-mediated dilation (FMD)) and muscle oxygen saturation $\left(\mathrm{StO}_{2}\right)$ parameters in pregnant women within a randomised, crossover, double-blind design in which twelve pregnant women consumed a single dose $(140 \mathrm{ml}$ ) of BJ or placebo (PLA). Urinary nitrate was assessed before (T0) and 150 min after BJ/PLA consumption. FMD was used to evaluate macrovascular endothelial function, and near-IR spectroscopy was used to evaluate muscle $\mathrm{StO}_{2}$ parameters during the occlusion and reperfusion phases, which were taken at baseline (PRE) and 120 and $140 \mathrm{~min}$ after BJ/PLA consumption, respectively. A significant increase in urinary nitrate was observed at $150 \mathrm{~min}$ after BJ consumption when compared with T0 (BJ: 0.20 (SD 0·13) $v$. T0: 0.02 (SD $0 \cdot 00), P=0.000$ ) and PLA intervention (PLA: 0.02 (sD 0.00), $P=0.001$ ). FMD improved after BJ consumption when compared with PRE (BJ: 11.00 (SD 1.67) $v$. PRE: 5.53 (sD 1.17), $P=0.000$ ) and PLA (5.34 (sD 1.31), $P=0.000$ ). No significant difference between PLA and PRE in FMD $(P=1.000)$ was observed. In $\mathrm{StO}_{2}$ parameters, a difference was not observed after BJ consumption compared with PRE and PLA intervention. The data demonstrate that a single dose of $140 \mathrm{ml}$ of $\mathrm{BJ}$ consumption improves macrovascular endothelial function, but not $\mathrm{StO}_{2}$ parameters.

Key words: Dietary nitrate: Functional food: Vascular function: Microcirculation: Pregnancy

During pregnancy, a reduction in macrovascular endothelial function (measured by flow-mediated dilatation (FMD)) is associated with hypertensive syndromes, whereas an increase in $\mathrm{FMD}$ reduces the risk of $\mathrm{CVD}^{(1)}$. In addition, preserved microvascular endothelial function in pregnancy is necessary for adequate tissue oxygenation. For example, impairments in uteroplacental vascular function in pregnancy lead to increased vascular resistance and decreased blood flow across the placenta, which reduce the placental capacity for nutrient and gas exchange $^{(2)}$. The low oxygen availability may compromise oxygen delivery to the fetus and favour the development of fetal growth restriction $(\mathrm{FGR})^{(3)}$. The NO molecule is critically involved in maintaining uteroplacental blood flow in pregnancies as NO regulates vascular tonus. Thus, the reduction of NO bioavailability has been associated with $\operatorname{FGR}^{(2,4,5)}$.

The regulation of vascular tonus varies according to anatomical location (conduit artery or resistance vessel) owing to different vascular morphologies ${ }^{(6)}$. Measuring FMD is a way of evaluating macrovascular endothelial function in the conduit artery (i.e. brachial artery), whereas microvascular function (microcirculation) may be evaluated by near-IR spectroscopy (NIRS), a technology that observes changes in tissue oxygen saturation $\left(\mathrm{StO}_{2}\right)$, allowing for the evaluation of tissue oxygen extraction (oxidative capacity) and microvascular function (tissue arterioles function) during the occlusion and reperfusion periods, respectively ${ }^{(7,8)}$.

Beetroot has gained the attention of the scientific community owing to its beneficial effects on cardiovascular health. The function of beetroot consumption on the risk of CVD has been studied and clinical trials have shown that beetroot is linked to improvement at both the macro-circulatory and the microcirculatory levels $^{(7-13)}$. Beetroot has beneficial effects on cardiovascular health owing to the nitrate present in this food. Nitrate can be converted to nitrite in the mouth by action of commensal facultative anaerobic bacteria that express nitrate reductase enzymes. In the acidic stomach and with low oxygen availability, nitrite is rapidly converted to $\mathrm{NO}^{(8,13-15)}$.

Effects of beetroot juice (BJ) consumption on macrovascular endothelial function have been studied, demonstrating improvements in endothelial function (by FMD) in elderly people with risk

Abbreviations: BJ, beetroot juice; FGR, fetal growth restriction; FMD, flow-mediated dilatation; GDM, gestational diabetes mellitus; NIRS, near-IR spectroscopy; PLA, placebo; PRE, baseline; $\mathrm{StO}_{2}$, oxygen saturation; $\mathrm{StO}_{2}$ base, baseline oxygen saturation; $\mathrm{StO}_{2}$ max, maximal oxygen saturation; $\mathrm{StO}_{2}$ min, minimum oxygen saturation; $\mathrm{StO}_{2}$ slope__1, desaturation rate; $\mathrm{StO}_{2}$ slope__2, resaturation rate; $\mathrm{StO}_{2}$ tmax , time to maximal oxygen saturation. 
factors for CVD (after ingestion of a single dose of beetroot-based nutritional gel containing approximately $12 \mathrm{mmol}$ nitrate $\left.^{(9)}\right)$; in healthy subjects after consuming $500 \mathrm{ml}$ of $\mathrm{BJ}^{(10)}$; in treated and untreated hypertensive subjects after $28 \mathrm{~d}$ of $\mathrm{BJ}$ consumption containing approximately $6.4 \mathrm{mmol}$ nitrate ${ }^{(11)}$; and in hypercholesterolaemic adult patients after 6 weeks of BJ consumption containing approximately $6 \mathrm{mmol}$ nitrate $^{(12)}$. The effects of $\mathrm{BJ}$ consumption at microcirculatory levels have also been evaluated by NIRS measurement. In healthy subjects, $6 \mathrm{~d}$ of ingestion of BJ containing approximately $0.07 \mathrm{mmol}$ nitrate $/ \mathrm{kg}$ body promoted an improvement in muscle oxygenation status during submaximal and maximal exercise in severe acute hypoxia ${ }^{(7)}$. Also in healthy subjects, $6 \mathrm{~d}$ of $500 \mathrm{ml}$ of BJ consumption containing $11.2 \mathrm{mmol}$ of nitrate promoted an improvement in indexes of muscle oxygenation during exercise ${ }^{(8)}$.

Although evidence assessing the effect of BJ consumption on macrovascular function and $\mathrm{StO}_{2}$ parameters exists, no study in pregnant women has been performed. Therefore, the present study investigated whether a single dose of BJ would improve FMD and $\mathrm{StO}_{2}$ parameters in pregnant women. A single dose of $\mathrm{BJ}$ was hypothesised to result in increased NO bioconversion, consequently promoting beneficial changes in FMD and $\mathrm{StO}_{2}$ parameters in pregnant women.

\section{Methods}

\section{Subjects}

This study was conducted in accordance with the guidelines set forth in the Declaration of Helsinki, and the research ethics committee of the Universidade Federal do Rio de Janeiro, Campus Macaé, Brazil (protocol no. 56732216.5.0000.5699), approved all procedures involving human subjects. The study was registered with the Clinical Trials Registry (NCT03315806). All pregnant women gave their written informed consent. A total of thirty-six pregnant women were recruited through announcements in flyers and advertisements during community events in the Macaé City, Rio de Janeiro Estate, Brazil; twentyfour recruits withdrew for personal reasons or were ineligible according to the inclusion criteria; and twelve recruits were randomised (Fig. 1). Pregnant women were selected if they were between 20 and 35 weeks of gestation and were over 18 years of age. Exclusion criteria included the presence of diabetes mellitus and gestational diabetes, hypertensive syndromes, HIV positive, liver or kidney disease, beetroot allergy, use of antioxidants and medication that alters endothelial function (angiotensin-converting enzyme inhibitor), use of antibiotics up to 2 weeks before the beginning of the study.

\section{Study design}

A randomised, double-blind, crossover and placebo (PLA)controlled study was conducted from October 2016 to May 2017. All subjects reported to the Dynamic Vascular Laboratory of Federal University of Rio de Janeiro, Macaé City, Brazil, on three occasions, with at least a 1-week interval between visits. The first visit was used to register basal (PRE) macrovascular and microvascular function measurements, blood sample collection for the characterisation of pregnant women, followed by

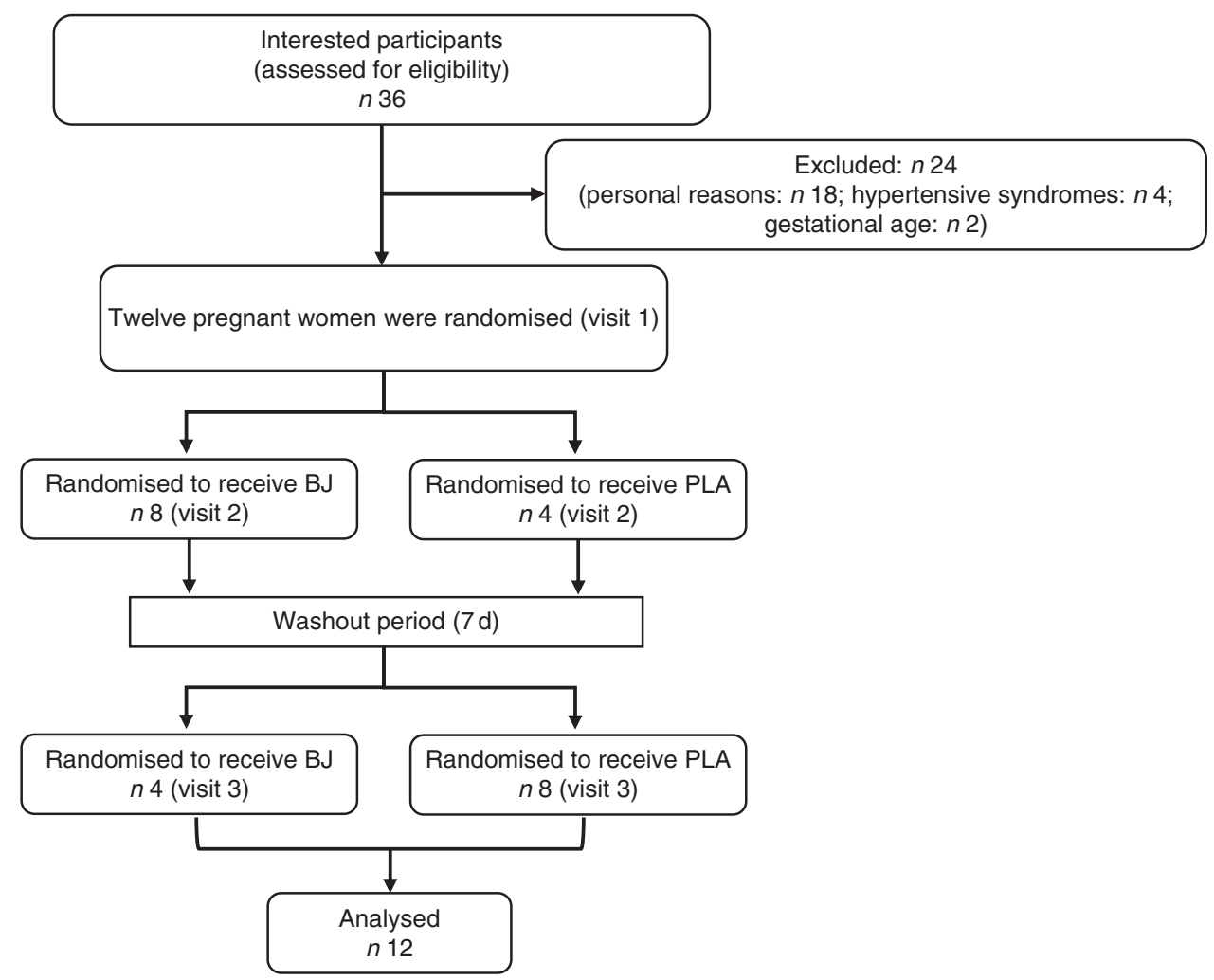

Fig. 1. Schematic of participant recruitment of the study. BJ, beetroot juice; PLA, placebo. 


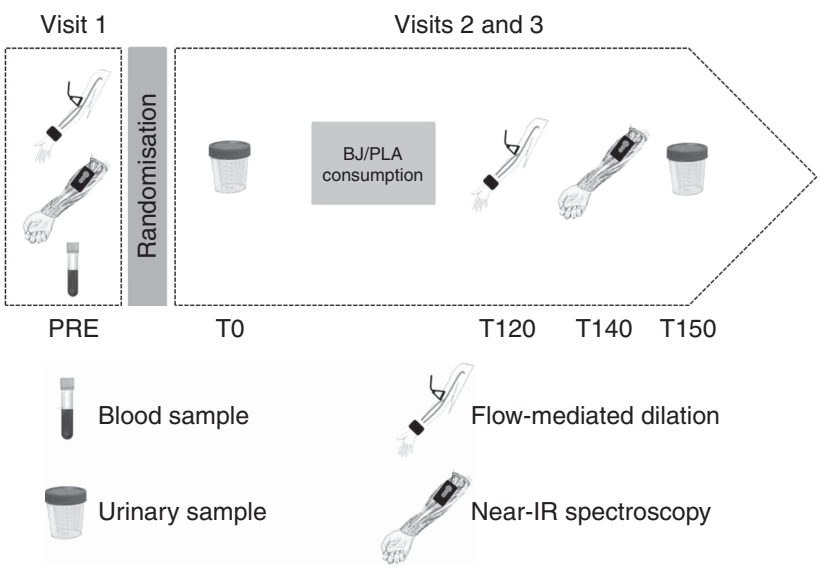

Fig. 2. Experimental design. BJ, beetroot juice; PLA, placebo.

randomisation (2:1 BJ:PLA). The $\mathrm{BJ}$ production requires several steps, including sanitation, centrifugation, pasteurisation and portioning. Production of small quantities of BJ for only one pregnant women was impracticable because it requires considerable time for production. In the randomisation $1: 1$, the remaining BJ would be lost as no preservatives were added, hence its short shelf life. Furthermore, it is important to point out that refrigerating (at $4{ }^{\circ} \mathrm{C}$ ) and freezing (at $-20^{\circ} \mathrm{C}$ ) the juice for 1 week could reduce the nitrate content by 15 and $14 \%$, respectively ${ }^{(16)}$. Thus, randomisation 2:1 (BJ:PLA) was more convenient in guaranteeing no nitrate losses. The participants were randomised and assigned by laboratory staff to ensure blind measurement. In the second and third visit, sample urine was drawn before (T0) BJ or PLA consumption. Afterward, macrovascular endothelial function, $\mathrm{StO}_{2}$ parameters and urine samples were collected, respectively, at $120 \mathrm{~min}$ (T120), $140 \mathrm{~min}$ (T140) and $150 \mathrm{~min}$ (T150) after BJ or PLA consumption (Fig. 2). The three visits were held between 07.00 and 12.00 hours. The participants were instructed to fast for at least $8 \mathrm{~h}$ before each visit; to restrict carbonated mineral water; to avoid the intake of foods rich in nitrate and nitrite; to not use any mouthwash; to not consume coffee or teas; and not to exercise for at least $12 \mathrm{~h}$ before the visits.

\section{Beetroot/placebo juice preparation}

The beetroots (Beta vulgaris L.) were washed in tap water, sanitised with a chlorine solution and centrifuged (Model CE700; Black \& Decker). The resulting juice was pasteurised, and $140 \mathrm{ml}$ of juice was distributed in two bottles of dark colour. For preparation of the PLA (control nitrate-depleted juice), the nitrate was removed from $\mathrm{BJ}$ by using a specific anionexchange resin A-520E (Purolite ${ }^{\circledR}$ ). The contents of nitrate of the BJ and PLA consumed by the subjects of this study were 8.95 (SD 0.28) and $0.27(\mathrm{SD} 0.02) \mathrm{mm} / 140 \mathrm{ml}$, respectively.

\section{Urinary nitrate}

Nitrate analysis in urine was performed as previously described by Croitoru $^{(17)}$ by using a HPLC system. A volume of $100 \mu \mathrm{l}$ of sample was added to $100 \mu \mathrm{l}$ of acetonitrile (v/v) and centrifuged
(10 min at $14000 \mathrm{rpm}$ ) for deproteinisation. Thereafter, $150 \mu \mathrm{l}$ of supernatant was mixed with $150 \mu \mathrm{l}$ of mobile phase $(\mathrm{v} / \mathrm{v})(5 \mathrm{~mm}$ tetrabutylammonium hydroxide) and the solution was mixed. Finally, $150 \mu \mathrm{l}$ was injected in HPLC. Sodium nitrate was used for quantification, and the results were expressed as $\mathrm{mmol} /$ mmol creatinine for nitrate.

\section{Macrovascular endothelial function analysis}

The evaluation of the FMD was performed in a quiet airconditioned room (constant temperature of $22-25^{\circ} \mathrm{C}$ ) $120 \mathrm{~min}$ (T120) after BJ/PLA consumption. All examinations were conducted by the same investigator. Pregnant women rested quietly with an angle of $90^{\circ}$ between the femur and the trunk. Endothelial function was assessed in accordance with Oliveira et $a l .{ }^{(9)}$. FMD is calculated as the percentage dilated at the maximum peak brachial artery diameter after cuff deflation, relative to maximum baseline brachial artery diameter (BBD). All analyses were conducted by a single investigator who was blinded to the participant and supplementation order. CV was intraday $5 \cdot 1$ (SD $0 \cdot 31$ ) \% and interday $5 \cdot 1$ (SD $0 \cdot 12) \%$.

\section{Oxygen saturation parameters analysis}

Changes in tissue $\mathrm{StO}_{2}$ were detected by a NIRS device (PortaMon; Artinis Medical Systems) that was placed on the flexor muscles of each subject's dominant forearm. After the $\mathrm{StO}_{2}$ signals had been stable for $30 \mathrm{~s}$, the cuff was rapidly inflated to suprasystolic levels (i.e. $250 \mathrm{mmHg}$ ), to obstruct blood flow to forearm muscles and a 5 -min arterial occlusion was performed. The reperfusion phase after cuff release was recorded for more 2 min. During all tests, the NIRS system was connected to a personal computer via Bluetooth for data acquisition $(10 \mathrm{~Hz})$, analogue-to-digital conversion and subsequent analysis of the raw data (i.e. no filter was used) using native software (Oxysoft version 2.1.6; Artinis Medical Systems). The software calculates absolute changes in light absorption at the different wavelengths $(750$ and $850 \mathrm{~nm})$ of oxyHb $\left(\mathrm{O}_{2} \mathrm{Hb}\right)$ and deoxyHb ( $\mathrm{HHb}$ ) using the spatially resolved spectroscopy method. $\mathrm{StO}_{2}$ was calculated as $\left(\mathrm{O}_{2} \mathrm{Hb} / \mathrm{O}_{2} \mathrm{Hb}+\mathrm{HHb}\right) \times 100$. The following NIRS parameters were considered for statistical analysis: baseline $\mathrm{StO}_{2}\left(\mathrm{StO}_{2}\right.$ base), which represents the average of $30 \mathrm{~s}$ of $\mathrm{StO}_{2}$ before arterial occlusion; desaturation rate $\left(\mathrm{StO}_{2}\right.$ slope_1 $)$, which corresponds to the slope of $\mathrm{StO}_{2}$ (the rate of change in $\mathrm{StO}_{2}$ per time) during the arterial occlusion phase; minimum $\mathrm{StO}_{2}\left(\mathrm{StO}_{2}\right.$ min), which corresponds to the lowest $\mathrm{StO}_{2}$ value during the arterial occlusion phase; resaturation rate $\left(\mathrm{StO}_{2}\right.$ slope_2 $)$, which corresponds to the slope of $\mathrm{StO}_{2}$ during the reperfusion phase; maximal $\mathrm{StO}_{2}\left(\mathrm{StO}_{2} \max \right)$, which corresponds to the highest $\mathrm{StO}_{2}$ value during the reperfusion phase; time to maximal $\mathrm{StO}_{2}\left(\mathrm{StO}_{2}\right.$ tmax $)$, which corresponds to the time to reach the $\mathrm{StO}_{2}$ max. $\mathrm{CV}$ was intraday 1.6 (SD 0.9) \% for baseline, 2.0 (SD 1.6) \% for occlusion and 1.6 (SD 1.1 ) \% for reperfusion and interday 1.8 (SD 0.2 ) \% for baseline, 2.9 (SD 0.7$) \%$ for occlusion and 1.5 (SD 0.1 ) \% for reperfusion. 


\section{Statistical analysis}

An a priori power analysis was conducted (G*Power version 3.0.1) for an $F$ test (ANOVA, repeated measures, within factors). On the basis of statistical power $(1-\beta)$ of $0 \cdot 80$, an effect size of 0.35 and an overall level of significance of 0.05 , at least sixteen participants were needed to detect a statistical difference. Although this study was unable to achieve the sample size of sixteen participants, the effect size was taken into account in order to determine the magnitude of the $\mathrm{BJ}$ ingestion effect on endothelial function and muscle $\mathrm{StO}_{2}$ parameters ${ }^{(18)}$. In addition, previous studies have observed statistical difference in $\mathrm{StO}_{2}$ parameters with $\leq 12$ volunteers after beetroot supplementation ${ }^{(8,9,19)}$.

The normality and homogeneity of variances of the data were examined with the Shapiro-Wilk and Mauchly tests, respectively, and were violated only for $\mathrm{StO}_{2}$ base. To identify differences in the FMD and $\mathrm{StO}_{2}$ parameters between PRE, PLA and BJ interventions, a repeated-measures oneway ANOVA was performed. When the assumption of normality and homogeneity of variances were violated, a Friedman test was used. To identify differences in urinary nitrate between BJ and PLA, a two-way ANOVA was used. In addition, ANOVA with repeated measurement was also performed to verify whether the order of BJ or PLA condition affected the results in this study. The effect size to observe the magnitude of the effect of $\mathrm{BJ}$ ingestion compared with PLA was calculated by Cohen's $d$, where a value $<0.2$ is considered trivial, $0.2-<0.5$ is a small effect, $0.5-<0.8$ is a moderate effect and $\geq 0.8$ is a large effect. Statistical significance was set at the 0.05 level of confidence. All analyses were performed using a commercially available statistical package (IBM SPSS Statistics version 23 for Mac) and the results were expressed as means and standard deviations.

\section{Results}

Of the thirty-six assessed for eligibility, twelve (33\%) were randomised and completed the study (Fig. 1). Furthermore, the $\mathrm{StO}_{2}$ data of one pregnant woman had to be excluded owing to technical reasons regarding the loss of NIRS signal. Therefore, only eleven volunteers were considered for analysis of this variable. The pregnant women were adult (27 (SD 6.51) years), sedentary, with a gestational age of 20-35 weeks, singleton pregnancies and the pregnant women did not use medications. The baseline volunteers' characteristics are shown in Table 1.

\section{Urinary nitrate}

T0 values of urinary nitrate $(\mathrm{mmol} / \mathrm{mmol}$ creatinine) were not different between groups (PLA: 0.02 (SD 0.00) v. BJ: 0.02 (sD 0.00), $P=0.866$ ). A significant main effect for time was observed $(P=0 \cdot 001)$. Post hoc test revealed that urinary nitrate increased 10-fold in T150 after BJ consumption compared with T0 (T150: 0.20 (sD 0.13) $v$. T0: 0.02 (sD 0.00), $P=0 \cdot 000)$, but not for PLA consumption compared with T0 (T150: 0.02 (SD 0.00) v. T0: 0.02 (SD 0.00), $P=0.992$ ). Furthermore, there was a significant treatment $\times$ time interaction effect for urinary nitrate $(P=0 \cdot 001)$. Post hoc test revealed that urinary nitrate increased 10-fold in T150 after BJ consumption compared with PLA $(P=0.001)$. In addition, BJ and PLA have a large effect size $(d=1.95)$.

\section{Endothelial function}

The endothelial function parameters are shown in Fig. 3 and Table 2. FMD (\%) increased 2-fold after BJ consumption (11.00 (SD 1.67)) compared with PLA (5.34 (SD 1.31)) and PRE (5.53 (sD 1.17)). BJ and PLA have a large effect size $(d=3 \cdot 77)$. There were no changes on BBD and PDB after BJ compared with PRE and PLA. In addition, there was no effect on the supplementation order $(\mathrm{BJ} \rightarrow \mathrm{PLA} v$. PLA $\rightarrow \mathrm{BJ})$ for FMD $(P=0.547)$, $\operatorname{BBD}(P=0.673)$ and $\operatorname{PDB}(P=0.658)$.

\section{Microvascular function}

The microvascular function parameters are shown in Fig. 4 and Table 3. There were no changes in $\mathrm{StO}_{2}$ base, $\mathrm{StO}_{2}$ slope-1, $\mathrm{StO}_{2}$ min, $\mathrm{StO}_{2}$ slope_2, $\mathrm{StO}_{2}$ max and $\mathrm{StO}_{2}$ tmax after $\mathrm{BJ}$ compared with PRE and PLA. In addition, there was no effect on the supplementation order (BJ $\rightarrow$ PLA $v$. PLA $\rightarrow \mathrm{BJ}$ ) for $\mathrm{StO}_{2}$ base $(P=0.784), \quad \mathrm{StO}_{2}$ slope-1 $(P=0.720), \mathrm{StO}_{2} \min (P=0.784)$, $\mathrm{StO}_{2}$ slope_2 $(P=0.518), \mathrm{StO}_{2} \max (P=0.671)$ and $\mathrm{StO}_{2} \mathrm{t}_{\max }$ $(P=0 \cdot 386)$. BJ/PLA have a trivial effect size for $\mathrm{StO}_{2}$ base $(d=0 \cdot 29), \mathrm{StO}_{2}$ slope_1 $(d=0 \cdot 03), \mathrm{StO}_{2}$ min $(d=0 \cdot 04), \mathrm{StO}_{2}$ slope_2 $(d=0 \cdot 04), \mathrm{StO}_{2} \max (d=0 \cdot 06)$ and $\mathrm{StO}_{2} \operatorname{tmax}(d=0 \cdot 03)$.

\section{Discussion}

The aim of this study was to examine the effects of a single dose of $\mathrm{BJ}$ on macrovascular endothelial function $\mathrm{FMD}$ and $\mathrm{StO}_{2}$

Table 1. Baseline demographic and clinical characteristics (Mean values and standard deviations)

\begin{tabular}{|c|c|c|}
\hline Variables & Mean & $\mathrm{SD}$ \\
\hline \multicolumn{3}{|l|}{ Demographics } \\
\hline$n$ & \multicolumn{2}{|c|}{12} \\
\hline Age (years) & 27 & $6 \cdot 51$ \\
\hline Gestational age (weeks) & $25 \cdot 6$ & $6 \cdot 24$ \\
\hline Body mass $(\mathrm{kg})$ & $79 \cdot 14$ & $20 \cdot 20$ \\
\hline Pre-pregnancy BMI $\left(\mathrm{kg} / \mathrm{m}^{2}\right)$ & $25 \cdot 41$ & $4 \cdot 81$ \\
\hline BMI $\left(\mathrm{kg} / \mathrm{m}^{2}\right)$ & $29 \cdot 3$ & $7 \cdot 04$ \\
\hline Forearm adipose tissue thickness (mm) & $2 \cdot 17$ & 0.83 \\
\hline \multicolumn{3}{|l|}{ Biochemistry } \\
\hline Total cholesterol (mmol/l) & 6.52 & 1.04 \\
\hline Glucose (mmol/l) & $4 \cdot 23$ & 0.54 \\
\hline HDL-cholesterol (mmol/l) & 1.58 & 0.34 \\
\hline LDL-cholesterol (mmol/l) & $4 \cdot 12$ & 0.87 \\
\hline TAG $(\mathrm{mmol} / \mathrm{l})$ & $1 \cdot 78$ & 0.37 \\
\hline TAG/HDL-cholesterol (mmol/l) & $1 \cdot 15$ & 0.28 \\
\hline Log(TAG/HDL-cholesterol) (mmol/l) & 0.05 & 0.10 \\
\hline \multicolumn{3}{|l|}{ Clinical } \\
\hline $\mathrm{SBP}(\mathrm{mmHg})$ & $102 \cdot 88$ & $6 \cdot 27$ \\
\hline $\mathrm{DBP}(\mathrm{mmHg})$ & $70 \cdot 00$ & $6 \cdot 63$ \\
\hline HR (beats per min) & 82.5 & 8.04 \\
\hline
\end{tabular}

SBP, systolic blood pressure; DBP, diastolic blood pressure; HR, heart rate. 
parameters in pregnant women. This is the first investigation into the effects of the single dose of $\mathrm{BJ}$ on $\mathrm{FMD}$ and $\mathrm{StO}_{2}$ parameters $\left(\mathrm{StO}_{2}\right.$ base, $\mathrm{StO}_{2}$ min, $\mathrm{StO}_{2}$ slope_1 $, \mathrm{StO}_{2} \max , \mathrm{StO}_{2}$ slope_2 and $\mathrm{StO}_{2}$ tmax $)$ in pregnant women. The major finding of this study is that a single dose of a BJ improves FMD in pregnant women.

FMD is a non-invasive test of endothelial function, and changes in FMD values provide important prognostic information $^{(20)}$. The FMD technique promotes increased blood flow and vasodilation owing to changes in shear stress, and this phenomenon is mediated by release of NO. Subjects with CVD have lower FMD values (endothelial dysfunction), whereas increased FMD decreases the risk of CVD ${ }^{(1)}$. Low FMD values in pregnancy are associated with health complications of the mother and fetus, such as pre-eclampsia and FGR. It has been shown that pregnant women who developed pre-eclampsia and FGR have shown FMD values of 3.5 and $6.2 \%$, respectively, whereas pregnant women who did not develop pre-eclampsia and FGR presented FMD values of $8 \%{ }^{(3)}$. The authors concluded that endothelial function is impaired in pregnant women who develop pre-eclampsia and FGR. In the present study, although the pregnant women did not develop pre-eclampsia and/or FGR, a low baseline FMD (5\%) was observed, suggesting impaired endothelial function. After BJ consumption, the FMD values increased from 5 to $11 \%$ (meaning a $110 \%$ of increase), thereby reaching FMD values similar to pregnant women who did not develop pre-eclampsia and $\mathrm{FGR}^{(3)}$.

The results of FMD in the present study are in accordance with previous findings ${ }^{(9,11,21)}$. Oliveira et $a .^{(9)}$ found

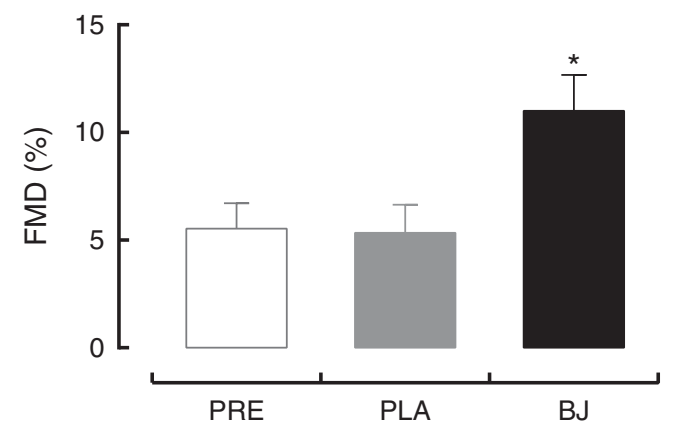

Fig. 3. Mean flow-mediated dilatation (FMD) after beetroot juice (BJ) and placebo (PLA) intervention. Values are means, with standard deviations represented by vertical bars. * Mean value was significantly different from baseline (PRE) and PLA $(P<0.001)$. improvements on FMD after ingestion of a single dose of beetroot-based nutritional gel rich in nitrate (containing approximately $12 \mathrm{mmol}$ nitrate in $100 \mathrm{~g}$ ) in the elderly with risk factors for CVD. Kapil et al. ${ }^{(11)}$ demonstrated increased FMD after $28 \mathrm{~d}$ of ingestion of $250 \mathrm{ml}$ of BJ (containing approximately $6.4 \mathrm{mmol}$ of nitrate in $250 \mathrm{ml}$ ) in hypertensive subjects. Asgary et $a l{ }^{(21)}$ observed improvements on FMD after 2 weeks of consumption of $250 \mathrm{ml}$ of $\mathrm{BJ}$ and $250 \mathrm{~g}$ of cooked beet in hypertensive subjects. Therefore, the results of these studies suggest that the effect of a single dose of BJ on FMD may be the same regardless of the population studied.

Increase in urinary nitrate is in accordance with previous findings ${ }^{(9,22)}$. Baião et al. ${ }^{(22)}$ demonstrated an increase in urinary nitrate concentration via bioconversion after ingestion of a single dose of $100 \mathrm{ml}$ of $\mathrm{BJ}$ (containing approximately $1.6 \mathrm{mmol}$ of nitrate). Oliveira et al. ${ }^{(9)}$ showed an increase in urinary nitrate concentration after ingestion of a single dose of beetroot-based nutritional gel rich in nitrate (containing approximately $12 \mathrm{mmol}$ nitrate in $100 \mathrm{~g}$ ). Siervo et al. ${ }^{(23)}$ showed an increase in nitrate concentration after ingestion of $140 \mathrm{ml}$ of BJ (containing $12 \mathrm{mmol}$ of nitrate) during $7 \mathrm{~d}$.

$\mathrm{StO}_{2}$ parameters during the arterial occlusion period $\left(\mathrm{StO}_{2}\right.$ min and $\mathrm{StO}_{2}$ slope-1) provide information about tissue oxygen extraction ${ }^{(24)}$. Dipla et al. ${ }^{(25)}$ evaluated recently $\mathrm{StO}_{2}$ parameters during arterial occlusion in pregnant women with gestational

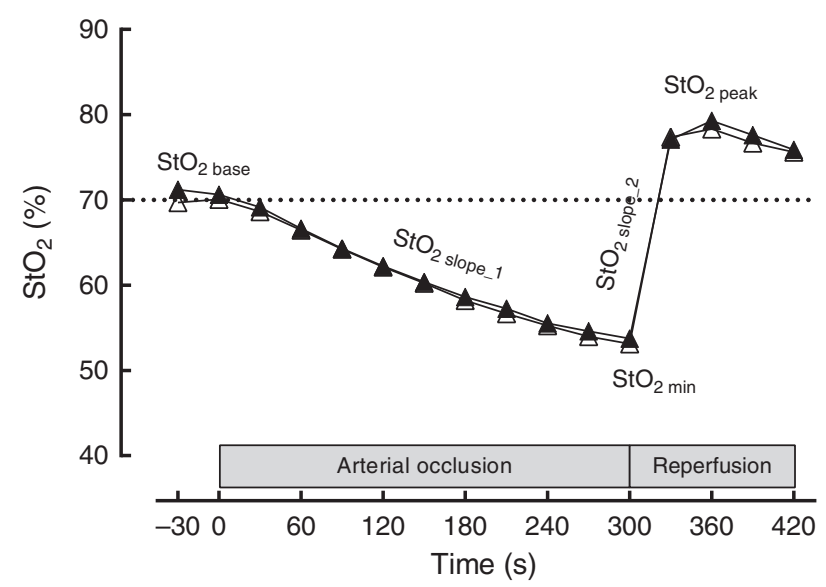

Fig. 4. Near-IR spectroscopy-derived profile of typical comportment of forearm muscle oxygen saturation $\left(\mathrm{StO}_{2}, \%\right)$ and the parameters evaluated. $\mathrm{StO}_{2}$ base, baseline oxygen saturation; $\mathrm{StO}_{2}$ slope_1, desaturation rate; $\mathrm{StO}_{2}$ slope_2, resaturation rate; $\mathrm{StO}_{2}$ min, minimum oxygen saturation. $\triangle$, Placebo; $\longrightarrow$, beetroot juice.

Table 2. Macrovascular endothelial function parameters at baseline and after beetroot juice (BJ) and placebo (PLA) consumption (Mean values and standard deviations)

\begin{tabular}{|c|c|c|c|c|c|c|}
\hline & \multicolumn{2}{|c|}{ PRE } & \multicolumn{2}{|c|}{ PLA } & \multicolumn{2}{|c|}{ BJ } \\
\hline & Mean & SD & Mean & SD & Mean & SD \\
\hline FMD (\%) & 5.53 & $1 \cdot 17$ & $5 \cdot 34$ & 1.31 & $11 \cdot 00^{\star \star \star}$ & 1.67 \\
\hline $\mathrm{BBD}(\mathrm{mm})$ & 3.39 & 0.63 & 3.34 & 0.50 & 3.28 & 0.66 \\
\hline PBD (mm) & 3.56 & 0.66 & 3.52 & 0.53 & 3.58 & 0.81 \\
\hline
\end{tabular}

PRE, baseline; FMD, flow-mediated dilation; BBD, maximum baseline brachial artery diameter; PBD, maximum peak brachial artery diameter.

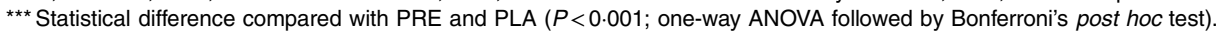


Table 3. Oxygen saturation $\left(\mathrm{StO}_{2}\right)$ parameters at baseline and after beetroot juice (BJ) and placebo (PLA) consumption*

(Mean values and standard deviations)

\begin{tabular}{|c|c|c|c|c|c|c|c|}
\hline & \multicolumn{2}{|c|}{ PRE } & \multicolumn{2}{|c|}{ PLA } & \multicolumn{2}{|c|}{ BJ } & \multirow[b]{2}{*}{$P$} \\
\hline & Mean & SD & Mean & SD & Mean & SD & \\
\hline $\mathrm{StO}_{2}$ base $(\%)$ & 71.39 & 8.97 & 69.69 & 4.25 & 71.19 & $5 \cdot 81$ & 0.336 \\
\hline $\mathrm{StO}_{2}$ slope_1 $(\% / \mathrm{s})$ & -0.52 & 0.30 & -0.60 & 0.32 & -0.58 & 0.29 & 0.908 \\
\hline $\mathrm{StO}_{2} \min (\%)$ & $54 \cdot 10$ & 13.03 & 52.83 & 12.99 & 53.37 & 12.41 & 0.973 \\
\hline $\mathrm{StO}_{2}$ slope_2 $(\% / \mathrm{s})$ & 1.03 & 0.65 & 1.03 & 0.55 & 1.06 & 0.72 & 0.993 \\
\hline $\mathrm{StO}_{2 \max }(\%)$ & 78.96 & 3.04 & 79.63 & $1 \cdot 18$ & 80.15 & 3.56 & 0.985 \\
\hline $\mathrm{StO}_{2 \operatorname{tmax}}(\mathrm{s})$ & $49 \cdot 28$ & $17 \cdot 84$ & 43.06 & 8.43 & 43.43 & 11.68 & 0.475 \\
\hline
\end{tabular}

diabetes mellitus (GDM). In GDM, the $\mathrm{StO}_{2}$ min value was greater when compared with the control group of pregnant women (approximately 55\% for GDM and $40 \%$ for control), suggesting that the GDM group exhibited a reduced capacity for $\mathrm{O}_{2}$ extraction at the skeletal muscle level. In the present study, pregnant women demonstrated an $\mathrm{StO}_{2}$ min value near the GDM group in the Dipla et $a l .{ }^{(25)}$ study, suggesting that the pregnant women in the present study possibly presented an impaired capacity for $\mathrm{O}_{2}$ extraction. It is already known that elevated blood glucose increases cell oxidative stress ${ }^{(26,27)}$, which impairs oxygen extraction by tissues (mitochondrial dysfunction) ${ }^{(28)}$. In this study, the pregnant women presented normal blood glucose $(<5.55 \mathrm{mmol} / \mathrm{l})$; however, they presented elevated cholesterol ( $>5 \mathrm{mmol} / \mathrm{l})$. Elevated blood cholesterol has also been shown to be associated with increased oxidative stress $^{(29)}$, which may impair oxygen extraction by tissues ${ }^{(26,27)}$. In addition, a significant correlation between $\mathrm{StO}_{2}$ min and cholesterol (date not showed) was observed, which may explain the greater $\mathrm{StO}_{2}$ min value in pregnant. It was expected that $\mathrm{BJ}$ consumption would improve $\mathrm{StO}_{2}$ parameters during arterial occlusion owing to the effect of nitrate on mitochondrial function. The nitrate-nitrite-NO pathway has been shown to modulate $\mathrm{O}_{2}$ consumption in mitochondria ${ }^{(30)}$. In complex IV of the electron transport chain, $\mathrm{NO}$ competes with $\mathrm{O}_{2}$ for cytochrome $c$ oxidase (COX), whereby NO binds to COX when oxygen is available ${ }^{(30)}$. However, no changes were observed in $\mathrm{StO}_{2}$ parameters during arterial occlusion. Bioconversion of nitrite to NO requires approximately $50 \% \mathrm{Hb}$-saturated oxygen $^{(31)}$. The present study observed that approximately $53 \% \mathrm{Hb}$ was saturated with oxygen, which indicates that the environment was favourable for nitrite-to-NO bioconversion, although no significant difference was observed in muscle oxygen extraction.

During the reperfusion phase, $\mathrm{StO}_{2}$ parameters $\left(\mathrm{StO}_{2}\right.$ max, $\mathrm{StO}_{2}$ slope-2 and $\mathrm{StO}_{2}$ tmax ) reflect the ability of recruiting greater arteriole numbers ${ }^{(32)}$. After arterial disocclusion, there is an increase in blood flow in the ischaemic limb, which promotes an increase in vasoactive substances ${ }^{(33)}$. An improvement in $\mathrm{StO}_{2}$ parameters after $\mathrm{BJ}$ consumption owing to vasodilator propriety of NO had been expected. However, there was no significant difference in $\mathrm{StO}_{2}$ parameters after $\mathrm{BJ}$ consumption.
The $\mathrm{StO}_{2}$ max and $\mathrm{StO}_{2}$ slope_2 in the present study was near the control group (healthy pregnant) values in Dipla et al. ${ }^{(25)}$ study, suggesting that pregnant women present a sufficient ability to recruit greater arteriole numbers. Hence, the integrity of microvascular reactivity in pregnant women in the present study may be a possible reason for the neglible effect on these parameters after BJ consumption. It was speculated that NO should be delivered only to the areas with impaired microvascular perfusion to avoid diffuse dilation in perfused capillaries. As microvascular reactivity is preserved in pregnant women of this study, the capillaries are sufficiently perfused and do not require increase in perfusion.

This study was unable to achieve a large sample size owing to physiological changes inherent in pregnancy, such as sea sickness and vomiting, as well as to the protocol of the study that requires periods of fasting. However, the strengths of the experimental study design are that all pregnant women were submitted to both BJ or PLA ingestion (crossover design), the muscle $\mathrm{StO}_{2}$ measure presented good repeatability (CV approximately $3 \%$ ), the pregnancies were within the gestational age of 20-35 weeks, the study included adults (27 (SD 6.51) years) and the ingestion of the food containing nitrate and nitrite before all experimental visits was controlled. In addition, the $\mathrm{BJ}$ ingestion is a potent nutritional supplement, which has been demonstrated to provoke changes in muscle $\mathrm{StO}_{2}$ parameters ${ }^{(34)}$. Therefore, the very small effect size reported in the present study suggests the weak magnitude of the BJ ingestion effect on muscle $\mathrm{StO}_{2}$ parameters ${ }^{(35)}$.

In addition, the differences among the responses observed in macro-circulatory and micro-circulatory levels after BJ consumption can be explained by cell heterogeneity. Differences in endothelial cells are reported at the level of morphology, function, gene expression and antigen composition ${ }^{(6)}$. Furthermore, endothelial cells exhibit differences depending on where the type of vessel or organ is located ${ }^{(36)}$. Therefore, NO action seems to be different in macrovasculature and microvasculature.

In summary, the effect of a single dose of BJ consumption, a source of dietary nitrate, on macrovascular endothelial function and $\mathrm{StO}_{2}$ parameters was investigated. The results of the present study show that $\mathrm{BJ}$ improves macrovascular endothelial function, but not $\mathrm{StO}_{2}$ parameters. Furthermore, BJ 
consumption increases urinary nitrate. The beneficial effect on macrovascular endothelial function observed after BJ consumption may be an important nutritional intervention for pregnant women aiming to reduce the risk of developing CVD. However, future studies are necessary to address and evaluate the effects of BJ consumption in pregnancy with CVD.

\section{Acknowledgements}

The authors thank Ricky Toledano for preparing the English version of the manuscript.

This work was supported by the Research Foundation of the State of Rio de Janeiro - FAPERJ (process no. E-26/111.297/ 2013 and E-26/110.309/2014). Funding was also provided from The Brazilian National Council for Scientific and Technological Development - CNPq (process no. 442977/2014-0).

M. V.-S. and G. V. d. O. contributed to volunteers' recruitment and physiological analysis. T. S. A. reviewed the manuscript. M. V.-S. contributed substantially to data acquisition, statistical analysis and data interpretation, and was the manuscript writer. All authors read and approved the final manuscript.

The authors have no conflicts of interest that are directly relevant to the content of this manuscript.

\section{References}

1. Green DJ, Jones H, Thijssen D, et al. (2011) Flow-mediated dilation and cardiovascular event prediction: does nitric oxide matter? Hypertension 57, 363-369.

2. Cottrell E, Tropea T, Ormesher L, et al. (2017) Dietary interventions for fetal growth restriction - therapeutic potential of dietary nitrate supplementation in pregnancy. $J$ Physiol 595 , 5095-5102.

3. Savvidou MD, Hingorani AD, Tsikas D, et al. (2003) Endothelial dysfunction and raised plasma concentrations of asymmetric dimethylarginine in pregnant women who subsequently develop pre-eclampsia. Lancet 361, 1511-1517.

4. Krause BJ, Carrasco-Wong I, Caniuguir A, et al. (2013) Endothelial eNOS/arginase imbalance contributes to vascular dysfunction in IUGR umbilical and placental vessels. Placenta 34, 20-28.

5. Chaddha V, Viero S, Huppertz B, et al. (2004) Developmental biology of the placenta and the origins of placental insufficiency. Semin Fetal Neonatal Med 9, 357-369.

6. Aird WC (2012) Endothelial cell heterogeneity. Cold Spring Harb Perspect Med 2, 1-3.

7. Masschelein E, Van Thienen R, Wang X, et al. (2012) Dietary nitrate improves muscle but not cerebral oxygenation status during exercise in hypoxia. J Appl Physiol (1985) 113, 736-745.

8. Bailey SJ, Winyard P, Vanhatalo A, et al. (2009) Dietary nitrate supplementation reduces the $\mathrm{O}_{2}$ cost of low-intensity exercise and enhances tolerance to high-intensity exercise in humans. J Appl Physiol (1985) 107, 1144-1155.

9. Oliveira GV, Morgado M, Pierucci AP, et al. (2016) A single dose of a beetroot-based nutritional gel improves endothelial function in the elderly with cardiovascular risk factors. J Funct Foods 26, 301-308.

10. Webb AJ, Patel N, Loukogeorgakis S, et al. (2008) Acute blood pressure lowering, vasoprotective, and antiplatelet properties of dietary nitrate via bioconversion to nitrite. Hypertension $\mathbf{5 1}$, 784-790.
11. Kapil V, Khambata RS, Robertson A, et al. (2015) Dietary nitrate provides sustained blood pressure lowering in hypertensive patients: a randomized, phase 2, double-blind, placebo-controlled study. Hypertension 65, 320-327.

12. Velmurugan S, Gan JM, Rathod KS, et al. (2016) Dietary nitrate improves vascular function in patients with hypercholesterolemia: a randomized, double-blind, placebo controlled study. Am J Clin Nutr 103, 25-38.

13. Bakker E, Engan H, Patrician A, et al. (2015) Acute dietary nitrate supplementation improves arterial endothelial function at high altitude: a double-blinded randomized controlled cross over study. Nitric Oxide 29, 58-64.

14. Lundberg JO, Gladwin MT, Ahluwalia A, et al. (2009) Nitrate and nitrite in biology, nutrition and therapeutics. Nat Chem Biol 5, 865-869.

15. Lundberg JO, Gladwin MT \& Weitzberg E (2015) Strategies to increase nitric oxide signalling in cardiovascular disease. Nat Rev Drug Discov 14, 623-641.

16. Corleto KA, Singh J, Jayaprakasha GK, et al. (2018) Storage stability of dietary nitrate and phenolic compounds in beetroot (Beta vulgaris) and arugula (Eruca sativa) juices. J Food Sci 83, 1237-1248.

17. Croitoru MD (2012) Nitrite and nitrate can be accurately measured in samples of vegetal and animal origin using an HPLC-UV/VIS technique. J Chromatogr B Analyt Technol Biomed Life Sci 911, 154-161.

18. Sullivan GM \& Feinn D (2012) Using effect size-or why the P value is not enough. J Grad Med Educ 4, 279-282.

19. Horiuchi M, Endo J, Dobashi S, et al. (2017) Muscle oxygenation profiles between active and inactive muscles with nitrate supplementation under hypoxic exercise. Physiol Rep 5, 1-6.

20. Harris RA, Nishiyama SK \& Wray DW. (2010) Ultrasound assessment of flow-mediated dilation. Hypertension 55, 1075-1085.

21. Asgary S, Afshani MR, Sahebkar A, et al. (2016) Improvement of hypertension, endothelial function and systemic inflammation following short-term supplementation with red beet (Beta vulgaris L.) juice: a randomized crossover pilot study. J Hum Hypertens 30, 627-632.

22. Baião D, Conte-Junior CA, Paschoalim VM, et al. (2016) Beetroot juice increase nitric oxide metabolites in both men and women regardless of body mass. Int J Food Sci Nutr $\mathbf{6 7}$, $40-46$.

23. Siervo M, Oggioni C, Jakovljevic DG, et al. (2016) Dietary nitrate does not affect physical activity outcomes in health older adults in a randomized, crossover trial. Nutr Res $\mathbf{3 6}$, 1361-1369.

24. Kolb L, Orbegozo D, Creteur J, et al. (2017) Oral nitrate increases microvascular reactivity and the number of visible perfused microvessels in healthy volunteers. J Vasc Res $\mathbf{5 4}$, 209-216.

25. Dipla K, Triantafyllou A, Grigoriadou I, et al. (2017) Impairments in microvascular function and skeletal muscle oxygenation in women with gestational diabetes mellitus: links to cardiovascular disease risk factors. Diabetologia 60, 192-201.

26. Asmat U, Abad K \& Ismail K. (2016) Diabetes mellitus and oxidative stress - a concise review. Saudi Pharm J 24, 547553.

27. Rochette L, Zeller M, Cottin Y, et al. (2014) Diabetes, oxidative stress and therapeutic strategies. Biochim Biophys Acta 1840, 2709-2729.

28. Pedersen BL, Baekgaard N \& Quistorff B. (2009) Muscle mitochondrial function in patients with type 2 diabetes mellitus and peripheral arterial disease: implications in vascular surgery. Eur J Vasc Endovasc Surg 38, 356-364. 
29. Rasool AH, Abdul Rahman AS, Abd Ghaffar NA, et al. (2010) Abnormal microvascular reactivity with hypercholesterolaemia in pregnancy. Malays J Med Sci 17, 14-19.

30. Larsen FJ, Schiffer TA, Borniquel S, et al. (2011) Dietary inorganic nitrate improves mitochondrial efficiency in humans. Cell Metab 13, 149-159.

31. Lundberg JO, Weitzberg E \& Gladwin MT (2008) The nitratenitrite-nitric oxide pathway in physiology and therapeutics. Nat Rev Drug Discov 7, 156-167.

32. Ryan TE, Brophy P, Lin CT, et al. (2014) Assessment of in vivo skeletal muscle mitochondrial respiratory capacity in humans by near-infrared spectroscopy: a comparison with in situ measurements. J Physiol 592, 3231-3241.
33. Oliveira GV, Morgado M, Conte-Junior CA, et al. (2017) Acute effect of dietary nitrate on forearm muscle oxygenation, blood volume and strength in older adults: a randomized clinical trial. PLOS ONE 12, 1-15.

34. Barry AE, Szucs LE, Reyes JV, et al. (2016) Failure to report effect sizes: the handling of quantitative results in published health education and behavior research. Health Educ Behav 43, 518-527.

35. Engelke KA, Halliwill JR, Proctor DN, et al. (1996) Contribution of nitric oxide and prostaglandins to reactive hyperemia in human forearm. J Appl Physiol (1985) 81, 1807-1814.

36. Potente M \& Mäkinen T (2017) Vascular heterogeneity and specialization in development and disease. Nat Rev Mol Cell Biol 18, 477-494. 